\title{
Hernia Repair during Cesarean Section
}

\section{-Compliance Matters: Tension-Free Tissue Repair}

\author{
Senol Carilli \\ General Surgery Department, American Hospital, Istanbul, Turkey \\ Email: carillis@yahoo.com
}

Received 2 December 2014; revised 17 December 2014; accepted 3 January 2015

Copyright (C) 2015 by author and Scientific Research Publishing Inc.

This work is licensed under the Creative Commons Attribution International License (CC BY).

http://creativecommons.org/licenses/by/4.0/

(c) (i) Open Access

\begin{abstract}
Purpose: Simultaneous surgeries are increasingly applied in all surgical fields. They bring cost and health advantages to the patient and the economy. Pregnancy is one of the risk factors of hernia in females. We conducted a study for simultaneous tissue repairs of inguinal and umbilical hernias during cesarean section. Methods: Between January 1997 and August 2013 we have operated 42 patients with 45 hernias either umbilical or inguinal. We performed the repairs from the Pfannenstiel incision. Data about maternal age, parity, cause of cesarean section, length of operation, length of stay, complications and patient satisfaction were collected. Results: Within this period there were 19,904 deliveries in total. Incidence of umbilical hernia was found $0.2 \%$ and it was $0.1 \%$ for the inguinal hernia. Simultaneous hernia repairs significantly prolonged the operative time. There was no recurrence and complication. Conclusions: Our results showed that hernia repair can be performed without any additional risks during cesarean section. We believe that if there is an indication for cesarean section in a pregnancy complicated by an abdominal wall hernia, patients should be informed that it would be safe and effective to repair these hernias during cesarean section. This approach also enables tension-free pure tissue repairs.
\end{abstract}

\section{Keywords}

Cesarean Section, Hernia Repair, Simultaneous Surgery, Tissue Repair, Abdominal Compliance

\section{Introduction}

In the 1980s, surgeons cautioned that simultaneous surgical treatment of more than one disorder was associated with increased risk and cumulative postoperative complication rates in these cases can be up to $50 \%$ higher than for single procedures [1]. Increased early postoperative morbidity was a concern in such situations, indeed, it has been suggested that combined hernioplasty and open prostatectomy may increase the risk of infection as a result 
of combining clean and contaminated surgeries [2]. Advances in anesthetic drugs and surgical techniques, particularly the introduction of minimally invasive methods, have made combined surgical procedures more popular. Today, it is not unusual for a second surgery to be piggy-backed on an elective procedure such as cholecystectomy, hernia repair, or prostatectomy.

Combined surgical procedures can be a modality of choice for improving cost effectiveness and medical results. Such patients require only one single hospital admission, one preoperative evaluation, one-time anesthesia, and have not a longer operative time, hospitalization time, and convalescence periods than two separate surgeries would demand [3] [4].

Mesh repairs are the standard for many hernias with very distinctive exceptions like Shouldice inguinal hernia repair. Actually, the standard is "tension free" repair if there is not a collagen metabolism abnormality.

Here, we reported prospectively collected series of patients undergoing concomitant cesarean section (CS) and umbilical or inguinal hernia tissue repairs those diagnosed during pregnancy.

\section{Methods}

Between January 1997 and August 2013, we prospectively investigated female patients at American Hospital General Surgery Department, who underwent elective CS and simultaneous inguinal and/or umbilical hernia repair, and compared to perioperative and early postoperative findings in a group of 100 randomly selected women who underwent CS alone. All the CSs were scheduled in the 36th to 39th weeks of pregnancy and all the hernias had been diagnosed during pregnancy. The procedures and risks involved were explained and each patient gave informed consent.

The data collected from all subjects were age, parity, indications for CS, single or multiple pregnancy, duration of surgery, type of anesthesia, intraoperative findings, perioperative and postoperative complications, and duration of hospital stay. For the CS-hernia patients, symptoms of the hernia and altered sensation at the hernia site such as numbness, tension and pain, after surgery were recorded as well. Duration of surgery was defined as the time elapsed from skin incision (beginning of the operation) to completion of the last skin suture. Patient satisfaction in hernia group was evaluated by questionnaire asking whether the individual would recommend this surgery to a friend. In each case, this was done twice: At hospital discharge and at the 1-month follow-up exam.

Just prior to the operation each woman received $1 \mathrm{~g}$ cefazolin sodium intravenously (IV). All CSs were performed by a gynecologist through a 10 to $14 \mathrm{~cm}$ Pfannenstiel incision at the pubic hairline. Once the skin and rectus fascia were opened, and dissected off a vertical peritoneal incision was made and the operation was performed in routine fashion. After the closure of uterus and bleeding control by gynecologists, the general surgeon started the hernia repair with a second gynecologist assisting.

For umbilical hernia repair, a Kocher clamp was placed on the middle of rectus abdominus sheet at the edge of the upper abdominal flap. The flap was retracted superiorly to expose the peritoneal aspect of the umbilical defect. A vertical incision was made in the peritoneum underlying the umbilicus and the caudal edge of the defect was identified easily by palpation. A second Kocher clamp was applied to this edge for traction and exposition of the defect. The assistant held the clamp in place while the surgeon approximated the edges of the fascia with a continuous pattern of 00 polypropylene suture, starting at least $1 \mathrm{~cm}$ caudal to the defect and ending $1 \mathrm{~cm}$ cranial to the superior edge of the defect (or a total of $4 \mathrm{~cm}$ from the starting point) then downward with the same suture for second row and ends are tied. Due to higher collagen content it is easy to palpate the caudal border of the umbilicus, whereas the cranial border is indistinctive due to attenuated collagen structure and not easily palpable. If the suture line is too short, a partial wall defect may remain which means missed hernia hence even if the defect cannot be palpated well we approximate a $4 \mathrm{~cm}$ part of the midline abdominal wall around the defect. Subsequently, peritoneal layer overlying the defect was closed.

For inguinal hernia repair, the rectus muscle was retracted up and the preperitoneal space was dissected laterally, extending through the internal inguinal ring. While the Pfannenstiel incision is horizontally proceeding through rectus abdominis sheet it leaves the muscle intact and enter the peritoneal cavity between left and right rectus abdominis muscles. At this level of the abdominal wall there is no posterior rectus sheet hence retraction of the muscle enable us to direct preperitoneal access to Retzius space then the space of Bogros.

After the identification of hernia and reduction of its content preperitoneal iliopubic tract repair was performed as described by Nyhus [5]. Continuous 00 polypropylene sutures were placed to close the posterior wall of the inguinal canal till to internal ring, and then a final 1 or 2 interrupted sutures were placed at the lateral edges of the ring. 
All CS-hernia patients were rechecked 1 week, 1 month, and then yearly after the combined procedures till to 5th years then phone call checks for yearly.

Means and standard deviations were calculated and group results were statistically compared using Student's $t$ test $(p=0.05)$.

\section{Results}

During the study period of 200 months 19,904 deliveries took place at our hospital and we performed CS + hernia repair in 42 of the mothers. Nine other pregnant women also had hernias (7 umbilical, 2 inguinal) but we were unable to perform hernia repair in these cases because the patients either delivered vaginally or had emergency CS. As well, 4 other pregnant women with hernias (3 umbilical, 1 inguinal) had repairs done by other surgeons who used different surgical techniques, and these cases were excluded from our study.

Of the 42 CS + hernia patients in the study, 24 had umbilical hernias, 15 had inguinal hernias, and 3 had both types. Of the inguinal hernias 10 were left sided and 8 were right. Two of the women had twin pregnancies and both had umbilical hernias only.

The overall incidence of umbilical hernias among the 19,904 total pregnancies during the study period was $0.2 \%(37 / 19,904)$ and that for inguinal hernia was $0.1 \%(21 / 19,904)$.

Of the 24 patients with umbilical hernias, 20 had combined epidural and spinal anesthesia patients and 4 had general anesthesia. All 15 patients with inguinal hernias had combined epidural and spinal anesthesia. Two of the 3 patients with both hernia types underwent combined epidural and spinal anesthesia, and the other had general anesthesia. All of the CS-hernia patients received epidural or IV patient-controlled analgesia in the postoperative period.

After the exclusion of 3 patients with combined umbilical and inguinal hernia the mean ages of the umbilical hernia group, the inguinal hernia group, and the control group were $32.5 \pm 3.17$ years, $35.1 \pm 3.61$ years, and $32.1 \pm 3.93$ years respectively (Table 1 ). The mean age of the inguinal hernia patients was significantly older than that of the others $(p=0.05)$. With regard to parity, para is significantly low in the control group.

The mean durations of surgery for the umbilical and inguinal hernia groups (respectively, $54.1 \pm 12.73 \mathrm{~min}$ and $53.9 \pm 5.8 \mathrm{~min}$ ) were significantly longer than the corresponding mean for the control group (44.8 $\pm 12.6 \mathrm{~min})$.

In the umbilical hernia group, the indications for CS were previous CS (20 patients), cephalopelvic disproportion (3 patients), and uterine myomectomy (1 patient). In the inguinal hernia group, the indications were previous CS (11 patients) and cephalopelvic disproportion (4 patients).

There were no perioperative or postoperative complications. There were no recurrences observed neither during first five years of physical examinations nor the yearly phone controls in all groups.

In all patients with inguinal hernias, findings during surgery were dilated internal inguinal ring and no identifiable peritoneal sac. In 4 of the inguinal hernia patients, an existing inguinal cord lipoma was identified and reduced, and it was not possible to evaluate a direct hernia space because of the (flabby) lacked muscle tone abdominal wall following CS. None of the women with umbilical hernias had a peritoneal sac as well. Five of the umbilical hernia patients had hernia lipomas and, in most cases, it was not possible to definitively identify the upper margin of the umbilical defects on palpation. Consequently, it was not possible to accurately measure most of these defects. Due to patient selection criteria all they were diagnosed during pregnancy and none of them were larger than $1.5 \mathrm{~cm}$ diameter (a finger tip breadth).

Table 1. Results for the hernia and control groups (data reported as mean and standard deviation). Three patients having umbilical and inguinal hernias are not included.

\begin{tabular}{cccc}
\hline \multirow{2}{*}{ Number of Patients } & Umbilical Hernia & Inguinal Hernia & Control Group \\
\cline { 2 - 4 } & $\mathrm{N}=24$ & $\mathrm{~N}=15$ & $\mathrm{~N}=100$ \\
\hline Age (years); Mean, (sd) & $32.5(3.17)^{\mathrm{a}}$ & $35.1(3.61)^{\mathrm{a}, \mathrm{b}}$ & $32.1(3.93)$ \\
Gravida & $1.88(0.8)$ & $1.86(1.27)$ & $1.86(1.27)$ \\
Para & $0.81(0.55)^{\mathrm{b}}$ & $0.71(0.46)^{\mathrm{b}}$ & $0.24(0.5)$ \\
Duration of Surgery(min) & $54.1(12.73)^{\mathrm{b}}$ & $53.9(5.8)^{\mathrm{b}}$ & $44.8(12.6)$ \\
Hospital Stay (day) & $3.07(0.47)$ & $3(0.4)$ & $3(0)$ \\
\hline
\end{tabular}

Abbreviations: ${ }^{\mathrm{a}}$ Statistical difference between umbilical and inguinal hernia patient groups $(p<0.05)$; ${ }^{\mathrm{b}}$ Statistical difference between control group $(p$ $<0.05$ ). 
In the first week post-surgery, none of the patients who underwent umbilical hernia repair experienced significant pain after the surgery but eleven did report different sensation or tenderness around the umbilicus when pressure was applied. None of the patients who underwent inguinal hernia repair described these sensations at the surgery site.

All the CS-hernia patients had positive responses to the questionnaire and all stated that they would recommend this combined surgery to friends.

\section{Discussion}

One of the earliest descriptions of simultaneous surgeries was made by Ferguson [6]. He had written that it was not uncommon in his practice at that time to remove the uterus, salpynx, appendix, or prostate during a herniotomy. Ferguson also pronounced increased intra-abdominal pressure as one of the causative factors for hernias [6]. During the last century, biological factors such as ultrastructural alterations in connective tissue and various anatomical tendencies have been shown in hernia etiology [7]. Although the factors that are commonly listed as causative for hernias are still mostly speculative, epidemiologic studies have confirmed that pregnancy is a risk factor for abdominal wall hernias. However, females do not develop more hernias than males, in spite of the increases in intra-abdominal pressure and great tension on abdominal wall seen in pregnancy. Bendavid pointed out that, for every woman with a hernia, at least 19 men require treatment for some type of hernia [8]. One of the exceptions about this distribution is adult umbilical hernia. Although the numbers are changing there is a female predominance occurring particularly in females older than 40 years, obese, diabetic and multiparous [9] [10]. Actually, many patients attribute the onset of umbilical herniation to pregnancy; furthermore multiple pregnancies often precede the development of an umbilical hernia. Incidence rates of both umbilical and inguinal hernias in this patient population were lower than non-pregnant population, which was probably originated from the younger patient groups, involved in this study.

Only 4 articles in the medical literature discuss hernia repair during CS. First one is a case report published in 1987 that describes the direct and femoral hernia repairs during a CS of the same patient [11]. Another article reported in 5 patients with inguinal hernias and 3 patients with umbilical hernias repaired during CS [12]. Ghnnam et al. published for 48 umbilical hernias repaired during CSs done within a 2-year period [13]. They used the same approach with us, transperitoneal access, and they used mesh repair if the defect was larger than 3 cm and detected 1 recurrence in 36 patients who underwent primary hernia repair. Recently, Gabriele et al. published 19 women with inguinal hernias and 9 with umbilical hernias that were repaired during CS [14]. They used mesh repair via periumbilical incision for the umbilical hernias and Lichtenstein repair via a Pfannenstiel incision.

Our results cannot be directly compared to previous findings owing to differences in patient selection criteria and the repair methods. Obviously, any additional procedure extends the duration of surgery, as our operative times demonstrate. But it has not changed the hospitalization period. Hospitalization period is affected from many parameters like cultures, patient's motivation to be discharged, and health insurance policies. At our center, the routine hospital stay after CS is 3 days, which was not changed following simultaneous hernia repairs.

Statistically, para is significantly higher in hernia groups than control and inguinal hernia patients are significantly older than other groups. Interpretations of these variations are mostly speculative and probably they were originated from the small number of the groups.

Recurrence rate is the most important parameter for assessing the success of hernia repair techniques. For umbilical hernia, primary repairs reported as having $42 \%$ short-term recurrence rate [10]. It is quite similar for the inguinal hernia tissue repairs. Not surprisingly, they were mostly replaced by mesh repairs in hernia surgery. Furthermore, primary repairs lead to considerable tissue tension and, therefore, tend to be associated with discomfort and disability, and have higher rates of complications and recurrence than mesh operations. However, we prefer primary repair to mesh techniques in this particular group of patients. Cesarean section is a bloody procedure, and after the baby has been evacuated and the uterus closed, there may still be some devitalized tissue in the surgical environment that could induce infection or endometriosis in the presence of prosthetic material. Furthermore, there is no tension on the flabby abdominal wall and these repairs don't cause pain and disability. Our results are verifying the importance of the tension free repair, which can be supplied without mesh implant only in this particular patient group. Absence of recurrence in our series may be the reflection of postpartum remodeling of the abdominal wall and its relation with relaxin, which required further investigations [15]. The 
motivation to conduct this study came from our observations that abdominal wall mechanics are disturbed by the stretching that occurs during pregnancy, and not rarely resulting architectural changes like rectus muscles diastasis. While the study was conducted we decided to use pure tissue repairs despite the justified prosthetics usage benefits. Our previous experiences taught us the higher compliance of the abdominal wall early in the postpartum period and further more as a result of skepticism we do not prefer to use prosthetic material even there is no evidence of its relation with endometriosis.

"Tension free" concept in hernia repairs is provided almost always by the prosthetics. Contribution of prosthetics to hernia repairs are clear and beyond any debates. On the other hand, abdominal wall compliance is the main determinant of tension of the wall and the intra abdominal pressure [16]. It is known that the most successful repairs can be performed in the most compliant abdomens. Abdominal wall compliance and its relation with volume and pressure studied by ascitic drainage can be attributed pregnancy and delivery [17].

It is estimated that groin hernia is associated with 1 of every 1000 to 3000 pregnancies [18]. Umbilical hernia is the second most frequent hernia after groin hernias in non-pregnant population [19]. Our result presented the hernia incidence is about $0.3 \%$ during pregnancy and umbilical hernias are almost two times more frequent than as inguinal hernias. According to the Swedish Hernia Register Koch pointed out a higher proportion of emergency operations was carried out in women than men (16.9\% vs. 5.0\%, respectively) [20]. They also reported that these operations led to bowel resections in $16.6 \%$ and $5.6 \%$ of cases, respectively. While the pregnancy is a true risk factor for female hernias, with awareness and a careful examination it may be diagnosed more frequently, and many female patients can be avoided from an emergency hernia surgery by the additional hernia repair during CS.

Due to rarity of the condition patient groups are unfortunately small in this study and that prevents impressive conclusions. The other weakness of the study is follow up. It is a well-known fact that 5-year period is not long enough for recurrences following hernia surgery and phone calls are always questionable for exact diagnosis. But due to phone contact we have not lost the patients. Progressive nature of the hernia draws attention of patients within a period of time due to pain or swelling at the hernia site. While there is no $100 \%$ success rate in any described repair techniques, it will not be a surprise if we have some recurrences in the future.

Our results show that combining hernia repair with CS is effective and causes lesser trauma to patients even though it is not part of the aims of this study, it is clearly cost effective. We believe any kind of hernia repair during CS is beneficial to prevent another and may be more complicated surgeries. We prefer direct approximation of the fascial edges with polypropylene sutures although it is contradictory to normal population in postpartum period it brings the advantage of tension free repair with non-mesh repair.

\section{References}

[1] Tagaya, N., Kasama, K., Suzuki, N., Taketsuka, S., Horie, K. and Kubota, K. (2005) Simultaneous Laparoscopic Treatment for Diseases of the Gallbladder, Stomach, and Colon. Surgical Laparoscopy Endoscopy \& Percutaneous Techniques, 15, 169-171. http://dx.doi.org/10.1097/01.sle.0000166972.23725.47

[2] Othman, I. and Abdel-Maguid, A.F. (2005) Combined Transurethral Prostatectomy and Inguinal Hernioplasty. Hernia, 14, 149-153. http://dx.doi.org/10.1007/s10029-009-0575-1

[3] Wadhwa, A., Chowbey, P.K., Sharma, A., Khullar, R., Soni, V. and Baijal, M. (2003) Combined Procedures in Laparoscopic Sugery. Surgical Laparoscopy Endoscopy \& Percutaneous Techniques, 13, 382-386. http://dx.doi.org/10.1097/00129689-200312000-00007

[4] Sasaki, A., Nitta, H., Otuska, K., Kimura, Y., Obuchi, T. and Wakabayashi, G. (2010) Concomitant Laparoscopic Splenectomy and Cholecystectomy. Surgical Laparoscopy Endoscopy \& Percutaneous Techniques, 20, 66-68. http://dx.doi.org/10.1097/SLE.0b013e3181d8493e

[5] Nyhus, L.M. (1995) The Preperitoneal Approach and Iliopubictract Repair of Inguinal Hernias. In: Nyhus, L.M. and Condon, R.E., Eds., Hernia, 4th Edition, J. B. Lippincott Company, Philadelphia, 153-177.

[6] Ferguson, A.H. (1908) Indications for Operation. In: The Technic of Modern Operations for Hernia, 2nd Edition, Cleveland Press, Chicago, 58-72.

[7] Condon, R.E. and Carilli, S. (1994) The Biology and Anatomy of Inguinofemoral Hernia. Seminars in Laparoscopic Surgery, 2, 75-85.

[8] Bendavid, R. (2001) Femoral Hernias in Females: Facts, Figures, and Fallacies. In: Bendavid, R., Abrahamson, J., Arregui, M.E., Flament, J.B., Phillips, E.H., Eds., Abdominal Wall Hernias: Principles and Management, Springer Verlag New York, 639-640. 
[9] Rutkow, I.M. and Robbins, A.W. (1993) Demographic, Classificatory, and Socioeconomic Aspects of Hernia Repair in the United States. Surgical Clinics of North America, 73, 413-426.

[10] Deysine, M. (2001) Umbilical Hernias. In: Bendavid, R., Abrahamson, J., Arregui, M.E., Flament, J.B. and Phillips, E.H., Eds., Abdominal Wall Hernias: Principles and Management, Springer Verlag, New York, 680-684.

[11] Altchek, A. and Rudick, J. (1987) Preperitoneal Herniorrhaphy: Adjunct to Cesarean Section. Obstetrics \& Gynecology, 70, 470-471.

[12] Ochsenbein-Kolble, N., Demartines, N., Ochsenbein-Imhof, N. and Zimmermann, R. (2004) Cesarean Section and Simultaneous Hernia Repair. 139, 893-895.

[13] Ghnnam, W.M., Helal, A.S., Fawzy, M., Ragab, A., Shalaby, H. and Elrefaay, E. (2009) Paraumbilical Hernia Repair during Cesarean Section. Annals of Saudi Medicine, 29, 115-118. http://dx.doi.org/10.4103/0256-4947.51798

[14] Gabriele, R., Conte, M., Izzo, L. and Basso, L. (2010) Cesarean Section and Hernia Repair: Simultaneous Approach. Journal of Obstetrics and Gynaecology Research, 36, 944-949. http://dx.doi.org/10.1111/j.1447-0756.2010.01283.x

[15] Sherwood, O.D. (2004) Relaxin's Physiological Roles and Other Diverse Actions. Journal of Endocrine Reviews, 25, 205-234. http://dx.doi.org/10.1210/er.2003-0013

[16] Goldman, J.M., Rose, L.S., Morgan, M.D.L. and Denison, D.M. (1996) Measurement of Abdominal Wall Compliance in Normal Subjects and Tetraplegic Patients. Thorax, 41, 513-518. http://dx.doi.org/10.1136/thx.41.7.513

[17] Papavramidis, T.S., Michalopoulos, N.A., Mistriotis, G., Pliakos, I.G., Kesisoglou, I.I. and Papavramidis, S.T. (2011) Abdominal Compliance, Linearity between Abdominal Pressure and Ascitic Fluid Volume. The Journal of Emergencies, Trauma, and Shock, 4, 194-197. http://dx.doi.org/10.4103/0974-2700.82205

[18] Augustin, G., Matosevic, P., Kekez, T., Majerovic, M. and Delmis, J. (2009) Abdominal Hernias in Pregnancy. Journal of Obstetrics and Gynaecology Research, 35, 203-211. http://dx.doi.org/10.1111/j.1447-0756.2008.00965.X

[19] Rutkow, I.M. (2003) Demographic and Socioeconomic Aspects of Hernia Repair in the United States in 2003. Surgical Clinics of North America, 83, 1045-1051. http://dx.doi.org/10.1016/S0039-6109(03)00132-4

[20] Koch, A., Edwards, A., Haapaniemi, S., Nordin, P. and Kald, A. (2005) Prospective Evaluation of 6895 Groin Hernia Repairs in Women. British Journal of Surgery, 92, 1553-1558. http://dx.doi.org/10.1002/bjs.5156 
Scientific Research Publishing (SCIRP) is one of the largest Open Access journal publishers. It is currently publishing more than 200 open access, online, peer-reviewed journals covering a wide range of academic disciplines. SCIRP serves the worldwide academic communities and contributes to the progress and application of science with its publication.

Other selected journals from SCIRP are listed as below. Submit your manuscript to us via either submit@scirp.org or Online Submission Portal.
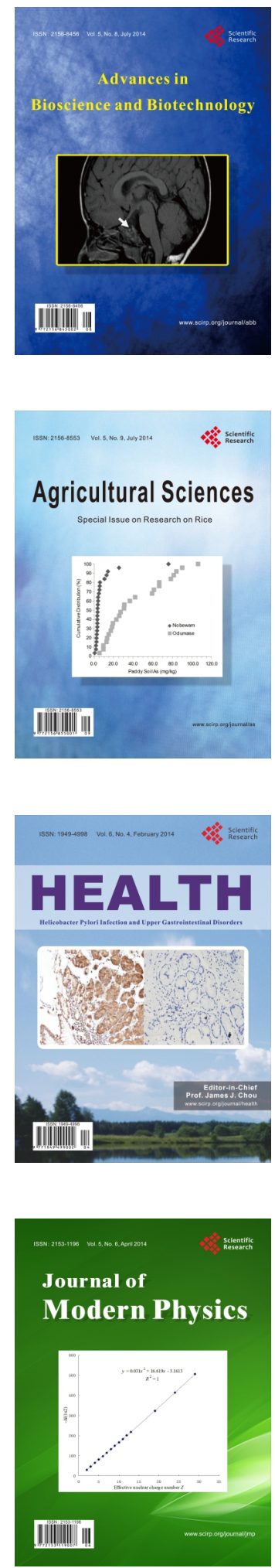
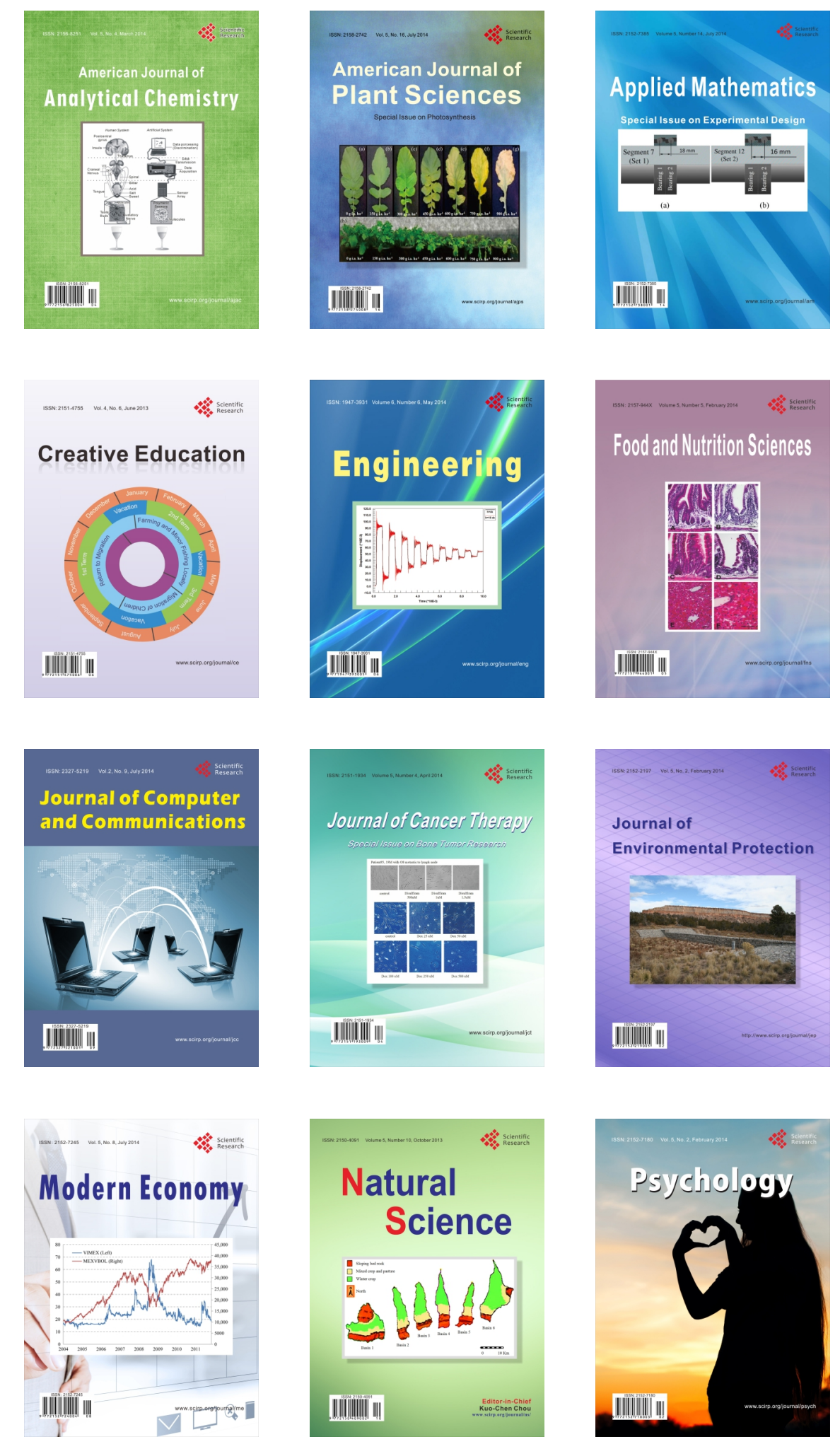